\title{
VIVENCIAS ACADÉMICAS DE LOS ESTUDIANTES DE I SEMESTRE MATUTINO DE TECNOLOGÍAS DE LA INFORMACIÓN-UNESUM
}

\author{
AUTORES: Luis Fernando Lucio Villacreses ${ }^{1}$ \\ Ing. Miriam Adriana Castillo Merino ${ }^{2}$ \\ Ing. José Efraín Álava Cruzatty ${ }^{3}$ \\ Ec. Robards Javier Lima Pisco ${ }^{4}$ \\ Dra. Yisell Vigoa Escobedo ${ }^{5}$
}

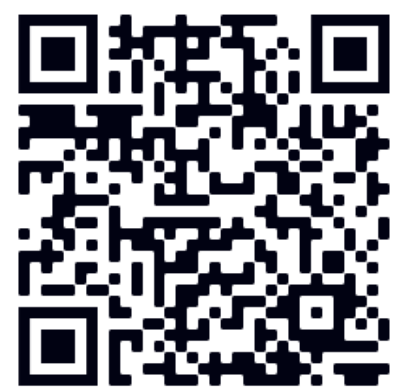

DIRECCIÓN PARA CORRESPONDENCIA:1uis.lucio@unesum.edu.ec

Fecha de recepción: junio29 de 2019

Fecha de aceptación: agosto 30 de 2019

\section{RESUMEN}

Con la finalidad de conocer las vivencias académicas de 74 estudiantes que cursan en horario matutino el primer semestrede la Carrera de Tecnologías de la Información y Comunicaciones de la Universidad Estatal del Sur de Manabí, se recurrió a la experiencia desarrollada en Colombia por la Universidad de Bogotá, donde se aplicó un cuestionario que permite conocer de los estudiantes: El relacionamiento personal e interpersonal que mantienen; su adaptación vocacional respecto a la carrera elegida; las competencias y hábitos de trabajo que mantiene dentro y fuera de la institución; y por último define el interés que tiene el estudiante hacia la institución donde estudia. Una vez aplicado el instrumento de medición, se pudo conocer que el $70,70 \%$ de los estudiantes gustan de la Universidad Estatal del Sur de Manabí, en contraste con el 29,30\% que unas veces están de acuerdo y otras veces no, en pertenecer a la institución, en este mismo contexto, el 39,10\% conoce los servicios que presta la universidad, mientras el 48,80\% algunas veces dice conocerlos y otras no. Respecto a la ubicación física de la carrera, el 58,50\% gusta del sector donde está situada. Todo lo expuesto, confluye en que el $63,50 \%$ de los estudiantes se plantee terminar la carrera que iniciaron y de este dato el 39,10\% piensaque la universidad posee una buena infraestructura. Finalmente, se debe señalar que este indicador se puede incrementar favorablemente si las áreas correspondientes realizan el mantenimiento y equipamiento continuo a todas las dependencias de la institución.

\footnotetext{
${ }^{1}$ Ing. Forestal, Magister en Educación y Desarrollo Social, Docente de la Carrera de Tecnologías de la Información de la Universidad Estatal del Sur de Manabí. Jipijapa, Manabí, Ecuador. 1uis.lucio@unesum.edu.ec

${ }^{2}$ Ing. Computación y Redes, Magister en Sistemas de Información Gerencial, Docente de la Carrera de Tecnologías de la Información de la Universidad Estatal del Sur de Manabí. Jipijapa, Manabí, Ecuador. adrianitacastime@gmail.com

${ }^{3}$ Ing.Telecomunicaciones, Magister en Telecomunicaciones, Docente de la Carrera de Tecnologías de la Información de la Universidad Estatal del Sur de Manabí. Portoviejo, Manabí, Ecuador. ingjosealava@gmail.com

${ }^{4}$ Economista, Magister en Administración Ambiental. Docente de la Carrera de Tecnologías de la Información de la Universidad Estatal del Sur de Manabí. Jipijapa, Manabí, Ecuador. robardslima@gmail.com

${ }^{5}$ Licenciada en Educación. Magister en Ciencias de la Educación. Doctora en Ciencias Pedagógicas. Universidad de Granma. Cuba.yvigoae@udg.co.cu
} 
Fernando Lucio Villacreses, Miriam Castillo M., José Álava Cruzatty, Robards Lima P., Yisell Vigoa Escobedo

Palabras clave:adaptación;interpersonal; personal; relacionamiento; vivencias.

\title{
ACADEMIC LIVES OF THE STUDENTS OF THE FIRST SEMESTER MATUTINE OF INFORMATION TECHNOLOGIES-UNESUM
}

\begin{abstract}
In order to learn about the academic experiences of 74 students who attend the first semester of the Information and Communications Technology Career at the State University of South Manabí in the morning, the experience developed in Colombia by the University of Bogotá, where a questionnaire was used to get to know the students: The personal and interpersonal relationships they allowed; their vocational adaptation regarding the chosen career; the skills and work habits that it maintains inside and outside the institution; and finally define the interest that the student has towards the institution where he studies. Once the measurement instrument was applied, it was possible to meet $70.70 \%$ of the students who like the South State University of Manabí, in contrast to the $29.30 \%$ who rarely agree and sometimes do not belong. to the institution, in this same context, 39.10\% know the services provided by the university, while $48.80 \%$ sometimes say they know them and others do not. Regarding the physical location of the race, $58.50 \%$ like the sector where it is located. All of the aforementioned, comes together in that $63.50 \%$ of the students consider finishing the degree that they started, and from this data $39.10 \%$ think that the university has a good infrastructure. Finally, it must indicate that this indicator can increase favorably if the corresponding areas maintain continuous maintenance to all the dependencies of the institution.
\end{abstract}

Keywords:adaptation; interpersonal; personal; relationship; experiences. 


\section{INTRODUCCIÓN}

La Constitución de la República del Ecuador (2008), en su artículo 26, estipula que "la educación es un derecho de las personas a lo largo de su vida y un deber ineludible e inexcusable del Estado" y, en su artículo 343, reconoce que el centro de los procesos educativos es el sujeto que aprende; por otra parte, en este mismo artículo se establece que "el sistema nacional de educación integrará una visión intercultural acorde con la diversidad geográfica, cultural y lingüística del país, y el respeto a los derechos de las comunidades, pueblos y nacionalidades".

La Ley Orgánica de Educación Intercultural, en el artículo 2, literal w): “Garantiza el derecho de las personas a una educación de calidad y calidez, pertinente, adecuada, contextualizada, actualizada y articulada en todo el proceso educativo, en sus sistemas, niveles, subniveles o modalidades; y que incluya evaluaciones permanentes. Así mismo, garantiza la concepción del educando como el centro del proceso educativo, con una flexibilidad y propiedad de contenidos, procesos y metodologías que se adapte a sus necesidades y realidades fundamentales. Promueve condiciones adecuadas de respeto, tolerancia y afecto, que generen un clima escolar propicio en el proceso de aprendizaje.".

La Universidad Estatal del Sur de Manabí-UNESUM, empeñada en profesionalizar a todos sus estudiantes, por una parte, crea ambientes de trabajo que cumplan los lineamientos establecidos por el Consejo de Aseguramiento de la Calidad de la Educación Superior-CACES, y por otra toma el modelo pedagógico constructivista y centra sus esfuerzos en lograr que cada docente genere procesos de enseñanza aprendizajeactivos, en el cual el estudiante cometerá errores y encontrará soluciones, muchas de estas a través de investigaciones planteadas en el aula o por necesidad propia del educando, quien se reconoce como autor de su propio aprendizaje y lo relaciona con su entorno inmediato, a través de experiencias, hechos y el entendimiento alcanzado, estructurando finalmente su conocimiento del mundo que le permite crear relaciones racionales y relaciones significativas.

Sin embargo, a pesar de lo expuesto anteriormente, se debe tomar en cuenta que el proceso de adaptación del estudiante en sus primeros inicios irá reflejando la distribución de su tiempo para: cumplir con horas clases previamente estipuladas, concretar trabajos autónomos que se planifican fuera del aula y participar en prácticas de enseñanza - aprendizaje, todo esto enmarcado en un entorno intensivo de formación. En este escenario, la relación: estudiantes - docentes - entorno educativo, visibilizará los estudiantes que están conforme con la carrera elegida y para aquellos que piensen lo contrario, será el momento para conocer las oportunidades que brinda la Carrera de Ingeniería en Tecnologías de la Información, frente a los retos de la globalización y la inmersión de las tecnologías emergentes en la sociedad en la que vivimos. Finalmente, se debe indicar la importancia de conocer las vivencias académicas del estudiante universitario, como una oportunidad para denotar sus apreciaciones frente a su relacionamiento personal e interpersonal, así como sus referencias respecto a su desenvolvimiento en la carrera que cursa, su forma de estudiar, y su postura frente a la institución universitaria. 
Fernando Lucio Villacreses, Miriam Castillo M., José Álava Cruzatty, Robards Lima P., Yisell Vigoa Escobedo

\section{DESARROLLO}

\section{Vivencias académicas}

El estudio denominado "Autoeficacia y vivencias académicasen estudiantes universitarios" realizado por (Borzone, 2017) quiendefine alas vivencias académicascomo las opiniones y sentimientos que el estudiante tiene respecto a experiencias cotidianas en el contexto de la enseñanza superior, y están compuestas por las siguientes variables: Dificultades personales. Angustia, desorientación, soledad, debilidad física, pesimismo, inestabilidad emocional, tristeza en oposición al desarrollo personal y la auto-confianza. Relaciones interpersonales. Las relaciones con compañeros de curso, de la universidad, de ambos sexos, o con amistades con mayor grado de intimidad (Mar Cornelio, et al. 2020), (Mar-Cornelio, et al. 2019).

- Valoración de la carrera, la identificación con la carrera elegida, el desarrollo de compromiso hacia esta, la adaptación y las perspectivas de desarrollo profesional acordes con aptitudes y capacidades. Estrategias de estudio. Las capacidades para estudiar, los hábitos de trabajo, la gestión del tiempo, el rendimiento obtenido, la utilización de la biblioteca y de otros recursos para el aprendizaje. Al respecto se señala también los siguientes aspectos importantes de saber:

$\checkmark$ Dimensión personal.-Involucra niveles adecuados dedisciplina, planificación, manejo del tiempo, tolerancia ala frustración y persistencia. Aquí, la adaptación universitariase asocia a la confianza en las propias capacidades, aldesarrollo de metas a seguir en el trabajo, al temperamentopositivo, al acceso a oportunidades y a los recursos necesariospara aprovecharlas.

$\checkmark$ Dimensión interpersonal.- Incluye las relaciones interpersonales, entendidas como procesos cognitivos, conductas y sentimientos experimentados por el estudiante en un contexto académico, los cuales pueden ser positivos si se asocian a la pertenencia, el reconocimiento y el bienestar.

$\checkmark$ Dimensión contextual.- Incluye aspectos de comunicación que emergen de las nuevas formas de relaciones pedagógicas y con los miembros de la institución en general, además de la relación del estudiante con la universidad, no como institución exclusivamente docente, sino como experiencia completa que facilita espacios tanto educativos como de esparcimiento y cuya oferta incluye, en ocasiones, servicios recreativos, espirituales y de salud física y mental.

$\checkmark$ Valoración de la carrera.- La identificación con la carrera elegida, el desarrollo de compromiso hacia esta, la adaptación y las perspectivas de desarrollo profesional acordes con aptitudes y capacidades.

$\checkmark$ Estrategias de estudio.- Las capacidades para estudiar, los hábitos de trabajo, la gestión del tiempo, el rendimiento obtenido, la utilización de la biblioteca y de otros recursos para el aprendizaje.

$\checkmark$ Valoración de la institución.- Interés por la universidad,el deseo de continuar los estudios y la valoración de suinfraestructura y de los servicios de apoyo de la misma.

98 UNESUM-Ciencias.Publicación cuatrimestral. Vol. III, Año 2019, No. 2 (agosto) 


\section{La deserción y el carácter de las metas individuales.}

Siempre habrá en una institución algunos estudiantes cuyas metas educativas son más limitadas o más amplias que las de la universidad a la que han ingresado. Para los alumnos con metas educativas restringidas, su actuación en la educación superior a menudo sólo implica acumular una cantidad determinada de créditos necesarios para certificaciones con fines profesionales, ascensos en el trabajo. Para estudiantes que trabajan medio tiempo, asistir a la universidad puede implicar el propósito de adquirir un conjunto de habilidades específicas (más bien que generales) requeridas por las tareas que desempeñan. Para estos estudiantes, como para otros, completar un programa de estudios puede no constituir un fin deseable; una corta asistencia a la universidad, en vez de la prolongada necesaria para terminar una carrera, puede resultar suficiente para lograr sus metas (Mar, Santana et al. 2019). Es posible encontrar la misma situación en aquellos alumnos cuyas metas educativas superan a las de la institución. En particular, un gran número de estudiantes puede entrar en instituciones con carreras de dos años con la intención de transferirse a otras universidades; han llevado a cabo lo que se proponían hacer en esa institución y, por lo tanto, la identificación de ese comportamiento con la deserción en su sentido estricto (es decir, como fracaso) es inexacto, porque tal denominación tergiversa sus intenciones, y engañosa, porque distorsiona el significado que ellos atribuyen a sus acciones(Tinto, 2000), (Mar and Gulín 2018) (Cornelio and Gulín 2018).

\section{Materiales y métodos.}

Para el desarrollo del presente trabajo, se partió de la investigación realizada por (S.Almeida, Gomes Ferreira, \& C.Soares, 1999) (Mar Cornelio, Gulín González et al. 2016), (Mar, Véliz et al. 2015) quienes elaboraron en su versión reducida el cuestionario de vivencias académicas en sus siglas definido como QVA-r, que tiene las siguientes dimensiones, descripciones e ítems:

\begin{tabular}{|c|c|c|c|}
\hline Dimensión & Descripción & $\begin{array}{l}\mathrm{N} .^{\circ} \mathrm{de} \\
\text { Ítems }\end{array}$ & Ítems que la conforman \\
\hline Personal & $\begin{array}{l}\text { Evalúa las percepciones del estudiante en } \\
\text { cuanto a bienestar físico y psicológico. }\end{array}$ & 13 & $\begin{array}{l}4,9,11,13,17,21,23,26 \\
28,39,45,52 \text { y } 55\end{array}$ \\
\hline Interpersonal & $\begin{array}{l}\text { Evalúa la relación con pares en general y } \\
\text { relaciones significativas, así como aspectos } \\
\text { relativos al involucramiento en actividades } \\
\text { extracurriculares. }\end{array}$ & 13 & $\begin{array}{l}1,6,19,24,27,30,33,36 \\
38,40,42,43 \text { y } 59\end{array}$ \\
\hline Carrera & $\begin{array}{l}\text { Evalúa la adaptación al pregrado, al } \\
\text { proyecto vocacional y las perspectivas de } \\
\text { carrera. }\end{array}$ & 13 & $\begin{array}{l}2,5,7,8,14,18,20,22,37 \\
51,54,56 \text { y } 60\end{array}$ \\
\hline Estudio & $\begin{array}{l}\text { Evalúa competencias de estudio, hábitos } \\
\text { de trabajo, organización del tiempo, uso } \\
\text { de la biblioteca y de otros recursos de } \\
\text { aprendizaje. }\end{array}$ & 13 & $\begin{array}{l}10,25,29,31,32,34,35 \\
41,44,47,49,53 \text { у } 57\end{array}$ \\
\hline Institucional & $\begin{array}{l}\text { Evalúa intereses hacia la institución, deseos } \\
\text { de continuar los estudios, conocimiento } \\
\text { y percepciones sobre la calidad de los } \\
\text { servicios y estructuras existentes. }\end{array}$ & 8 & $\begin{array}{l}3,12,15,16,46,48,50 \\
\text { y } 58\end{array}$ \\
\hline
\end{tabular}

CUniversidad Estatal del Sur de Manabí. Jipijapa, Ecuador. 


\section{Tabla 1. Caracterización psicométrica del QVA-r}

El cuestionario final quedó de la siguiente manera:

\begin{tabular}{|c|c|c|}
\hline PREGUNTA & DIMENSIÓN & DESCRIPCIÓN \\
\hline 4 & \multirow{13}{*}{ 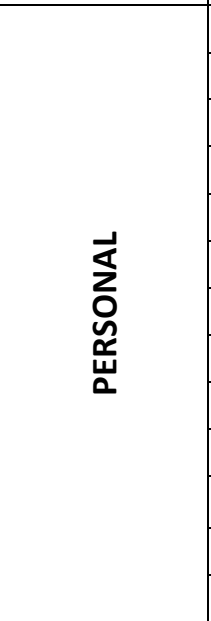 } & Presento cambios en mi estado de ánimo (inestabilidad emocional) \\
\hline 9 & & Me siento triste o abatido (a) \\
\hline 11 & & Últimamente me he sentido confundido (a) y desorientado (a) \\
\hline 13 & & Hay situaciones en la que siento que pierdo el control \\
\hline 17 & & Últimamente me sentido pesimista \\
\hline 21 & & Siento cansancio y somnolencia durante el día \\
\hline 23 & & Siento confianza en mi mismo (a) \\
\hline 26 & & Me he sentido solo durante el último tiempo (meses o semestre) \\
\hline 28 & & Tengo momentos de angustia \\
\hline 39 & & Pienso en muchas cosas que me ponen triste \\
\hline 45 & & Me siento físicamente débil \\
\hline 52 & & Tengo dificultad para tomar decisiones \\
\hline 55 & & Me he sentido ansioso (a) \\
\hline 1 & \multirow{13}{*}{ 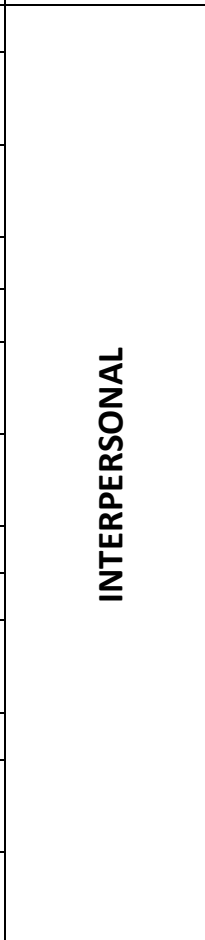 } & Hago amistades con facilidad en mi universidad \\
\hline 6 & & $\begin{array}{l}\text { Habitualmente me encuentro poco acompañado por otros } \\
\text { compañeros de la universidad }\end{array}$ \\
\hline 19 & & $\begin{array}{l}\text { Mis compañeros de curso son importantes para mi crecimiento } \\
\text { personal }\end{array}$ \\
\hline 24 & & Siento que tengo un buen grupo de amigos en la universidad \\
\hline 27 & & He establecido buenas relaciones con mis compañeros de curso \\
\hline 30 & & $\begin{array}{l}\text { Se me dificulta encontrar un compañero (a) de estudio que ayude a } \\
\text { resolver un problema personal }\end{array}$ \\
\hline 33 & & $\begin{array}{l}\text { Tengo relaciones de amistad cercana con compañeros de universidad } \\
\text { de ambos sexos }\end{array}$ \\
\hline 36 & & Se me facilita iniciar una conversación con nuevos compañeros \\
\hline 38 & & Se me conoce como una persona amigable y simpática \\
\hline 40 & & $\begin{array}{l}\text { Procuro compartir con mis compañeros de curso fuera de los horarios } \\
\text { de clase }\end{array}$ \\
\hline 42 & & Tomo la iniciativa para invitar a mis amigos a salir \\
\hline 43 & & $\begin{array}{l}\text { Mis relaciones de amistad son cada vez más estables, duraderas e } \\
\text { independientes }\end{array}$ \\
\hline 59 & & $\begin{array}{l}\text { Se me dificulta establecer relaciones cercanas con mis compañeros de } \\
\text { curso }\end{array}$ \\
\hline 2 & \multirow{6}{*}{ 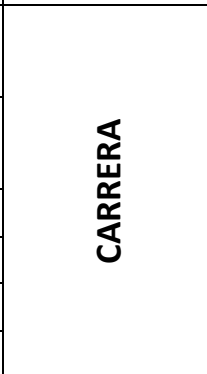 } & $\begin{array}{l}\text { Creo que puedo desarrollar nuevos aspectos personales (prestigio, } \\
\text { estabilidad, solidaridad) por medio de la carrera que elegí. }\end{array}$ \\
\hline 5 & & $\begin{array}{l}\text { Cuando pienso en mi pasado, consigo identificar las razones que me } \\
\text { llevaron a elegir esta carrera. }\end{array}$ \\
\hline 7 & & Elegí bien la carrera que estoy estudiando \\
\hline 8 & & Tengo capacidades y vocación para el área profesional que elegí \\
\hline 14 & & Me siento comprometido (a) con la carrera que elegí. \\
\hline 18 & & Las notas que he obtenido han sido buenas y me ha permitido avanzar \\
\hline
\end{tabular}

100 UNESUM-Ciencias.Publicación cuatrimestral. Vol. III, Año 2019, No. 2 (agosto) 
UNESUM-Ciencias: Revista Científica Multidisciplinaria VIVENCIAS ACADÉMICAS DE LOS ESTUDIANTES DE I SEMESTRE MATUTINO DE TECNOLOGÍA DE LA INFORMACIÓN

\begin{tabular}{|c|c|c|}
\hline & & en mi carrera. \\
\hline 20 & & $\begin{array}{l}\text { Cuento con capacidades de aprendizaje para responder a mis } \\
\text { expectativas en el estudio }\end{array}$ \\
\hline 22 & & $\begin{array}{l}\text { Creo que la carrera que elegí me permite desarrollarme } \\
\text { profesionalmente. }\end{array}$ \\
\hline 37 & & Elegí la carrera de acuerdo con mis aptitudes y capacidades \\
\hline 51 & & Me siento decepcionado de mi carrera. \\
\hline 54 & & Mis gustos personales fueron decisivos para elegir mi carrera \\
\hline 56 & & Estoy estudiando una carrera diferente a la que siempre quise \\
\hline 60 & & Aunque me fuera posible no me cambiaría de carrera \\
\hline 10 & \multirow{13}{*}{$\begin{array}{l}\frac{0}{0} \\
\frac{2}{2}\end{array}$} & Organizo bien mi tiempo \\
\hline 25 & & Me siento a gusto con un alto ritmo de mi trabajo \\
\hline 29 & & Utilizo la biblioteca de la universidad \\
\hline 31 & & Se me dificulta concentrarme en una tarea durante mucho tiempo \\
\hline 32 & & Planifico las actividades que debo realizar diariamente \\
\hline 34 & & Mantengo al día mis deberes académicos \\
\hline 35 & & $\begin{array}{l}\text { Mi incapacidad para organizar bien el tiempo hace que tenga bajo } \\
\text { desempeño académico }\end{array}$ \\
\hline 41 & & $\begin{array}{l}\text { Sé establecer prioridades con respecto a las actividades en las que } \\
\text { invierto mi tiempo }\end{array}$ \\
\hline 44 & & Logro tomar buenos apuntes en las clases \\
\hline 47 & & Siento que voy a lograr ser eficaz en la preparación de mis exámenes \\
\hline 49 & & Procuro organizar la información obtenida en las clases \\
\hline 53 & & Tengo buenas capacidades para el estudio \\
\hline 57 & & Soy puntual para llegar al inicio de las clases \\
\hline 3 & \multirow{8}{*}{ 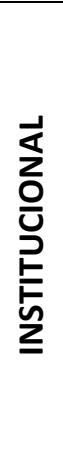 } & Aunque fuera posible no me cambiaría de universidad \\
\hline 12 & & Me gusta la universidad en la que estudio \\
\hline 15 & & Conozco bien los servicios que presta mi universidad \\
\hline 16 & & $\begin{array}{l}\text { Me gustaría terminar mis estudios en la universidad en que me } \\
\text { encuentro actualmente }\end{array}$ \\
\hline 46 & & Mi universidad no me genera interés \\
\hline 48 & & $\begin{array}{l}\text { La biblioteca de la universidad se encuentra bien dotada (cantidad y } \\
\text { variedad de bibliografía) }\end{array}$ \\
\hline 50 & & Me gusta el sector donde está ubicada mi universidad \\
\hline 58 & & Mi universidad posee buena infraestructura \\
\hline
\end{tabular}

Tabla 2: Cuestionario para medir las vivencias académicas

\section{Unidades de estudio.}

Las unidades de estudio de la presente investigación fueron los paralelos A y B horario matutino de primer semestre de la Carrera de Ing. en Tecnologías de la Información de la UNESUM, periodo académico noviembre 2018 - marzo 2019, en los que se tuvo una población de 74 estudiantes, con un promedio de edad de18 años. En el siguiente cuadro se amplían algunos aspectos de interés del presente trabajo: 


\begin{tabular}{|c|c|c|c|}
\hline Paralelo & Masculino & Femenino & Total \\
\hline A & 26 & 12 & 38 \\
\hline B & 24 & 12 & 36 \\
\hline TOTAL & 50 & 24 & 74 \\
\hline$\%$ & $68 \%$ & $32 \%$ & $100 \%$ \\
\hline
\end{tabular}

Tabla 3. Conformación de género

Elaborado por: Ing. Luis Fernando Lucio Villacreses

Los datos que se muestran a continuación fueron tomados del acta final de calificaciones de la asignatura de matemáticas discretas:

\begin{tabular}{|c|c|c|c|c|}
\hline \multirow{2}{*}{ Estado actual } & \multicolumn{2}{|c|}{$\begin{array}{c}\text { Número de } \\
\text { estudiantes según } \\
\text { paralelo }\end{array}$} & \multicolumn{2}{c|}{$\begin{array}{c}\text { Porcentaje de } \\
\text { estudiantes según } \\
\text { paralelo }\end{array}$} \\
\cline { 2 - 5 } & $\mathbf{A}$ & $\mathbf{B}$ & $\mathbf{A}$ & $\mathbf{B}$ \\
\hline Retirados & 2 & 2 & $5,56 \%$ & $5,26 \%$ \\
\hline Perdió semestre & 1 & 1 & $2,78 \%$ & $2,63 \%$ \\
\hline $\begin{array}{c}\text { Pérdida de créditos } \\
\text { promoviantes que fueron } \\
\text { semestre }\end{array}$ & 8 & 10 & $22,22 \%$ & $26,32 \%$ \\
\hline $\begin{array}{c}\text { Total (estudiantes que } \\
\text { cursaron primer semestre) }\end{array}$ & $\mathbf{3 6}$ & 25 & $69,44 \%$ & $65,79 \%$ \\
\hline
\end{tabular}

Tabla 4. Estatus actual de los estudiantes al término del período académico noviembre 2018 marzo 2019

Elaborado por: Ing. Luis Fernando Lucio Villacreses

102 UNESUM-Ciencias.Publicación cuatrimestral. Vol. III, Año 2019, No. 2 (agosto) 


\section{Resultados encontrados en la investigación.}

\section{Dimensión personal.}

Se observa que existe una tendencia en los estudiantes a sentir cambios en su estado de ánimo, lo que influye en generar confusiones, pesimismo, angustia y ansiedad, sin embargo, es evidente que un 48,80\% de los estudiantes se tienen confianza en sí mismos, lo que posibilitó que las emociones descritas no fueran mayores, promoviendo a su vez que los estudiantes se mantuvieran asistiendo a clases de forma normal. Es importante destacar que cada uno de los docentes que formaron parte del Periodo Académico noviembre 2018 - marzo 2019 han sido parte del proceso de adaptación de los estudiantes para discernir la importancia de la Carrera de Ingeniería en Tecnologías de la Información y para motivar al estudiantado frente al reto de profesionalizarse en mediano plazo.

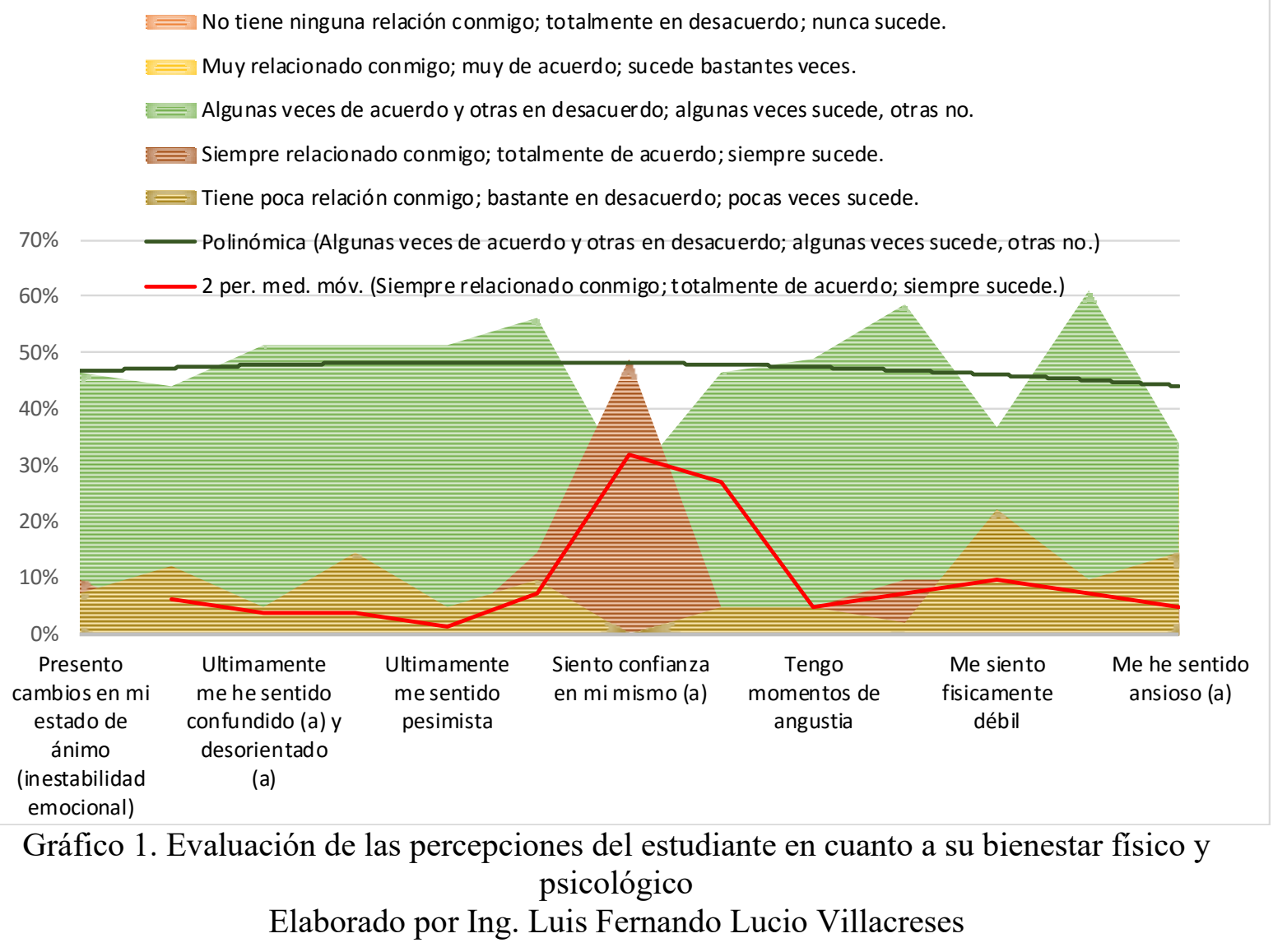


Fernando Lucio Villacreses, Miriam Castillo M., José Álava Cruzatty, Robards Lima P., Yisell Vigoa Escobedo

\section{Dimensión interpersonal:}

Se observa que los alumnos tienen buenas relaciones de amistad entre sí, volviéndose un coloquio de ayuda mutua, para tratar aspectos personales y asuntos académicos, este último, requiere la cooperación de los estudiantes que dominan contenidos para ayudar a sus compañeros que poseen dificultados de aprendizajes. Con esto se logra que los estudiantes realicen trabajos de docencia, trabajo autónomo y prácticas de aprendizaje,que promueven la resolución de problemas de la vida cotidiana. Otro de los factores que promueve vínculos entre los estudiantes, es la generación de proyectos de aula y la elaboración de Proyectos Integradores de Saberes, que invita a compartir experiencias entre los grupos participantes, docentes, y finalmente con un tribunal de sustentación que observa el crecimiento personal de los estudiantes y el compañerismo existente para afrontar los retos que el estudiante asume con responsabilidad.

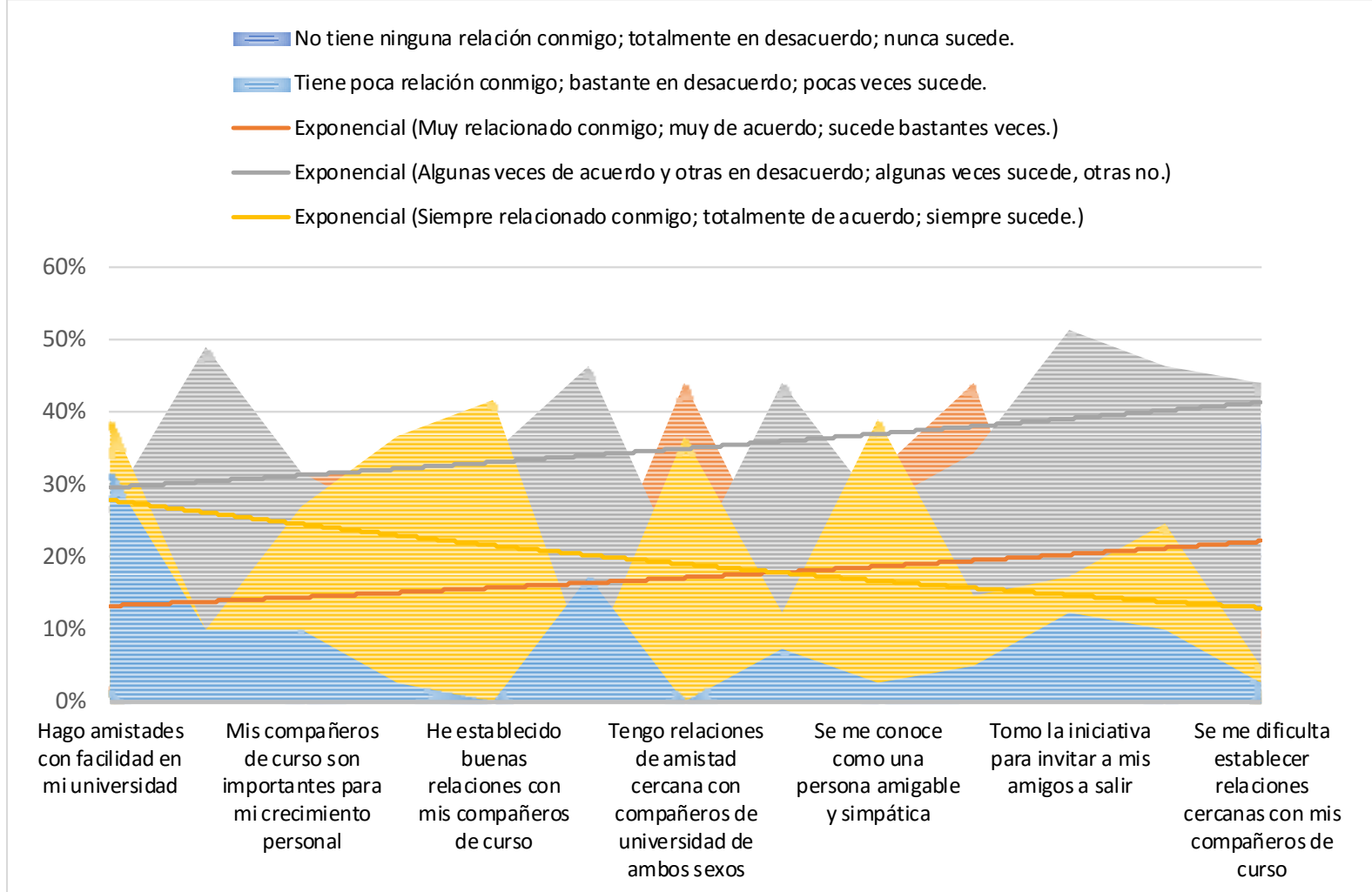

Gráfico 2.Evaluación de las relaciones interpersonales de los estudiantes que cursaron el primer semestre horario matutino de la Carrera de Ingeniería de Tecnologías de la Información. Elaborado por Ing. Luis Fernando Lucio Villacreses

En esta parte finalmente se debe indicar que los estudiantes han demostrado tener facilidad para realizar amistades con estudiantes de otras carreras de la UNESUM, así también, se evidencia que algunas veces sucede y otras no, lo cual permite que el estudiante este preparado para intentos fallidos en la generación de amistades durante su estancia académica en la universidad a

104 UNESUM-Ciencias.Publicación cuatrimestral. Vol. III, Año 2019, No. 2 (agosto) 
la que pertenece, esto habitualmente sucede con el $48,80 \%$ de los estudiantes, motivo del presente estudio.

\section{Dimensión carrera de estudio:}

Todos los estudiantes creen que pueden desarrollar nuevos aspectos personales como prestigio a través de la carrera que eligió estudiar, lo que esta en debate por no ser precisamente la opción inicialmente deseada, sin embargo, conforme se fue cursando el semestre académico noviembre 2018 - marzo 2019, se notó la generación de un compromiso individual del estudiante, basado en sus actitudes y capacidades de aprendizaje para responder a sus expectativas en la carrera elegida. Se debe señalar además que altérmino del semestre un $97,60 \%$ de los estudiantes, aunque les fuera posible no se cambiarían de carrera.

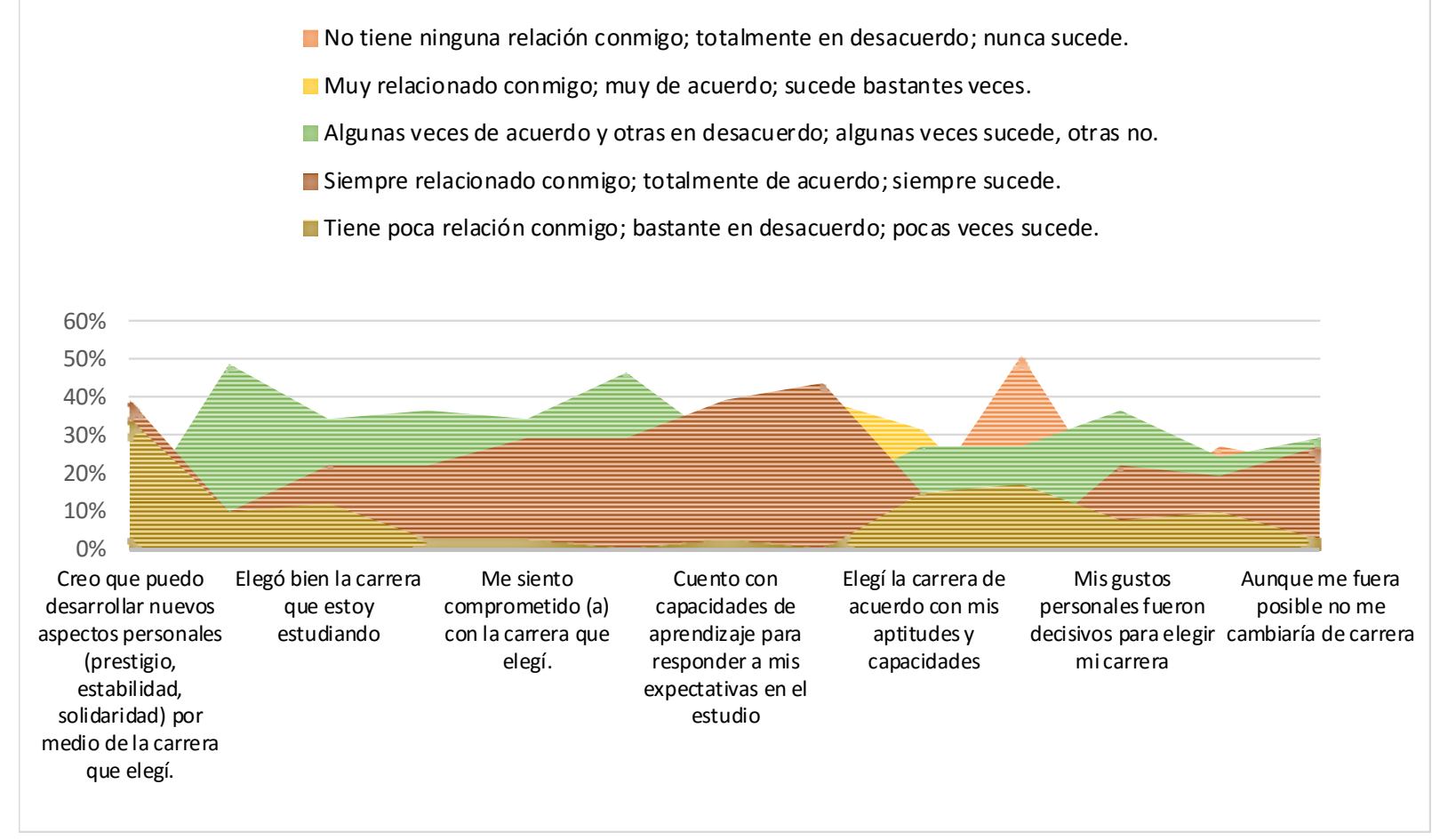

Gráfico 3. Adaptación al proyecto vocacional de los estudiantes que cursaron el primer semestre horario matutino de la Carrera de Ingeniería de Tecnologías de la Información.

Elaborado por Ing. Luis Fernando Lucio Villacreses

A pesar de lo expuesto en la parte superior se debe señalar que existe un 4,90\% de los estudiantes que se sienten decepcionados de su carrera.

\section{Dimensión estudio:}


Fernando Lucio Villacreses, Miriam Castillo M., José Álava Cruzatty, Robards Lima P., Yisell Vigoa Escobedo

Respecto al cumplimiento de las competencias, hábitos de trabajo, organización del tiempo, etc., prima en un $56,10 \%$ de los estudiantes el hecho de algunas veces organizar su tiempo y otras veces no, mientras el $34,10 \%$ siempre lo organiza bien. La misma tendencia se mantiene respecto al uso de la biblioteca de la universidad, en este caso existe un promedio del 53,70\% que alguna vez suele asistir, mientras el $17,10 \%$ no se relaciona con el uso de la biblioteca, esto seguramente ocurre por el hecho de que esta entidad no se encuentre dentro del predio donde está ubicada la Carrera de Ingeniera de Tecnologías de la Información.

Siguiendo con el análisis de este apartado, se aprecia un hábito de estudio en el cual el $63,40 \%$ de los discentes toman buenos apuntes en clases, organizan la información recibida por el docente y son puntuales al llegar al horario de clases. Lo expuesto tiene mucha relación con el porcentaje promedio de estudiantes que fueron promovidos al semestre superior, los cuales representan el $67,62 \%$ de la población en estudio.

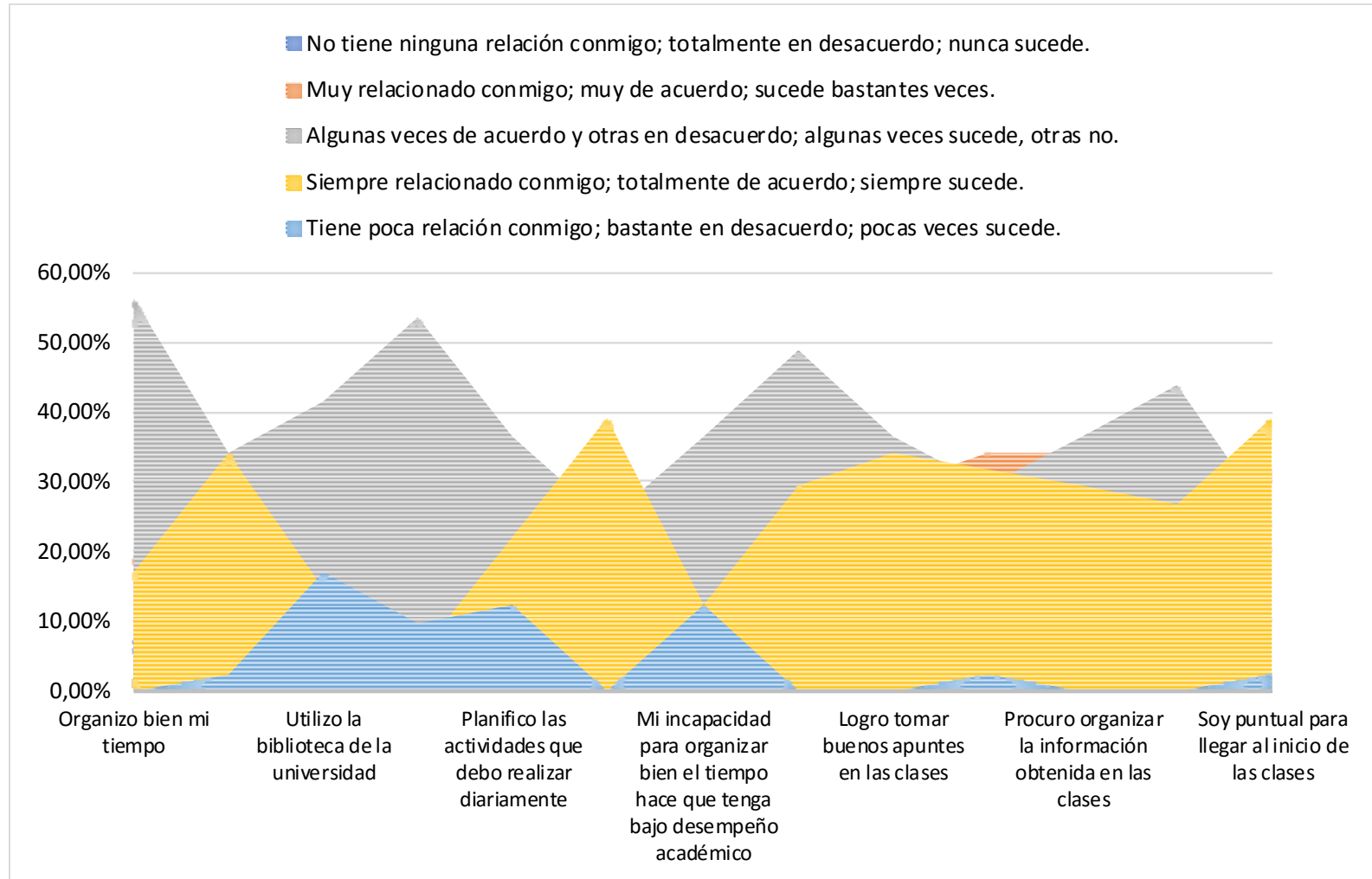

Gráfico 4. Evaluación de las competencias de los estudiantes que cursaron el primer semestre horario matutino de la Carrera de Ingeniería de Tecnologías de la Información.

Elaborado por Ing. Luis Fernando Lucio Villacreses

\section{Dimensión institucional:}

Respecto al dimensionamiento institucional se tiene una opinión del 70,70\% de los estudiantes quienes gustan de la Universidad Estatal del Sur de Manabí, en contraste con el 29,30\% que unas veces están de acuerdo y otras veces no, en este contexto, el 39,10\% conoce los servicios que

106 UNESUM-Ciencias.Publicación cuatrimestral. Vol. III, Año 2019, No. 2 (agosto) 
presta la institución y el 48,80\% algunas veces si y otras no. Respecto a la ubicación de la carrera de estudio, el 58,50\% le gusta el sector donde está situada. Todo lo expuesto confluye en que el $63,50 \%$ de los estudiantes se plantee terminar la carrera que iniciaron y de ellos un $39,10 \%$ piensan que la universidad posee una buena infraestructura.

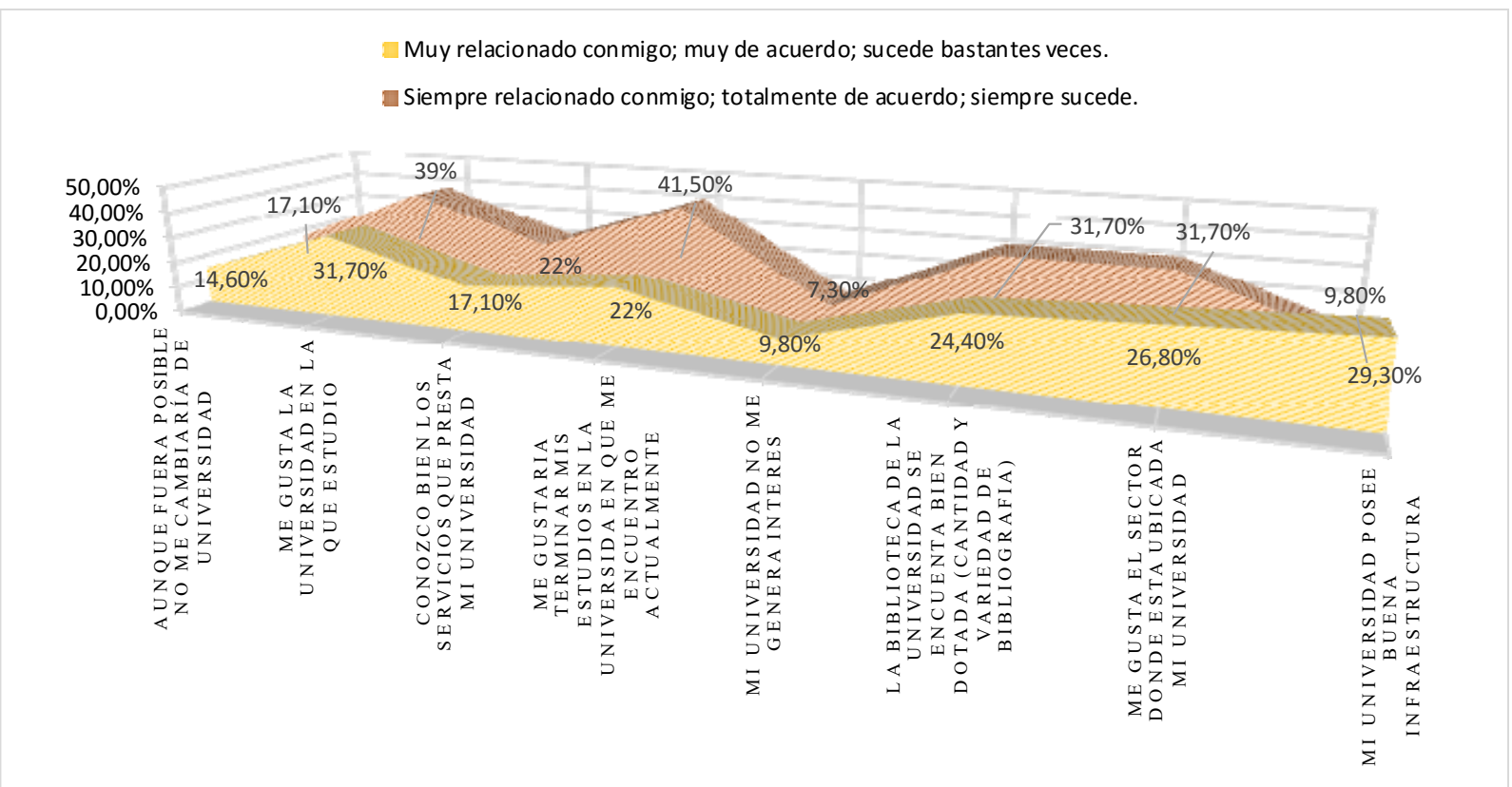

Gráfico 5. Evaluación de dimensión institucional por parte de los estudiantes que cursaron el primer semestre horario matutino de la Carrera de Ingeniería de Tecnologías de la Información. Elaborado por Ing. Luis Fernando Lucio Villacreses

\section{CONCLUSIONES}

Es evidente que la Universidad Estatal del Sur de Manabí posee una buena infraestructura, actualmente valorada por los estudiantes que cursan la Carrera de Ingeniería en Tecnologías de la Información, sin embargo, este indicador puede incrementar favorablemente si se da mantenimiento de forma continua a toda la infraestructura, y se mejora la climatización de las aulas y se amplia la cobertura de internet en cada una de sus áreas.

Se evidencia una débil atención delos docentes de primer semestre hacia sus dirigidos, por ende, no se transmite información total sobre los servicios que presta la carrera y la universidad, particularmente aquellos que buscan el bienestar de la comunidad estudiantil, como: el acceso a servicios de salud, ayudas económicas o becas por rendimiento académico y movilidad. En este cometido también se debe orientar que las fuentes de información oficial se alojan dentro de la página web de la universidad.

Es necesario potenciar la experiencia de cada estudiante en el manejo de la información y documentación,labor que desarrolla durante el semestre académico y se evidencia en un 
Fernando Lucio Villacreses, Miriam Castillo M., José Álava Cruzatty, Robards Lima P., Yisell Vigoa Escobedo

portafolio. Este trabajo debe ser reconocidos dentro del recorrido académico como un proceso de investigación, trabajo autónomo y prácticas de aprendizaje, que en términos de puntuaciones ganadas deben ser un incentivo para los discentes y un ejemplo para aquellos que no realizan un mayor esfuerzo. A esto se suma el habito de estudio que va optando el estudiante como un factor de éxito durante su formación profesional, todo en su conjunto puede sumar a la reducción de deserciones y la pérdida de las asignaturas que se cursan.

La encuesta aplicada puede ser utilizada para toda la colectividad universitaria a fin de conocer las apreciaciones de todos los estudiantes en torno a las dimensiones estudiadas y el crecimiento institucional de la Universidad Estatal del Sur de Manabí.

\section{REFERENCIASBIBLIOGRÁFICAS}

Borzone Valdebenito, M. A. (2017). Autoeficacia y vivencias académicas en estudiantes universitarios. Universidad de Concepción, Chile, 266-274.

Cornelio, O. M. and J. G. Gulín (2018). "Modelo para la evaluación de habilidades profesionales en un Sistema de Laboratorios a Distancia." Revista Cientifica 3(33): 1.

Márquez Rodríguez, D. P., Órtiz Urazán, S. C., \& Rendón Arango, M. (2009). Cuestionario de Vivencias Académicas en su versión reducida (QVA-r): un análisis psicométrico. REVISTA COLOMBIANA DE PSICOLOGÍA, 33-52.

Mar-Cornelio, O., I. Santana-Ching and J. González-Gulín (2019). "Sistema de Laboratorios Remotos para la práctica de Ingeniería de Control." Revista científica(36): 356-366.

S.Almeida, L., Gomes Ferreira, j. A., \& C.Soares, A. P. (1999). Cuestionario de vivenvias académicas: Construcción y validación de una versión reducida (QVA-r). Revista Portuguesa de Pedagogía, 181-207.

Mar Cornelio, O., J. Gulín González, I. Santana Ching and L. Rozhnova (2016). "Sistema de Laboratorios a Distancia para la práctica de Control Automático." Revista Cubana de Ciencias Informáticas 10(4): 171-183.

Tinto, V. (2000). DEFINIR LA DESERCION: UNA CUESTION DE PERSPECTIVA. Obtenido de www.comie.org.mx/congreso/memoriaelectronica/v11/docs/area_16/1799.pdf: http://publicaciones.anuies.mx/pdfs/revista/Revista71_S1A3ES.pdf

Mar Cornelio, O., I. Santana Ching and J. Gulín González (2020). "Operador por selección para la agregación de información en Mapa Cognitivo Difuso." Revista Cubana de Ciencias Informáticas 14(1): 20-39.

Mar, O. and J. Gulín (2018). "Model for the evaluation of professional skills in a remote laboratory system." Revista científica 3(33): 332-343.

Mar, O., I. Santana and J. Gulín (2019). "Algorithm to determine and eliminate neutral nodes in Cognitive Neutrosophic Map." Neutrosophic Computing and Machine Learning 8: 4-11.

Mar, O., Y. Z. Véliz, M. d. R. C. Felipe and M. L. Vázquez (2015). "Motor de inferencia decisional en sistema informático para la evaluación del desempeño." Revista Cubana de Ciencias Informáticas 9(4): 16-29. 